\title{
O CÂNCER BUCAL NO ESTADO DO RIO GRANDE DO NORTE: UM ESTUDO ECOLÓGICO
}

Oral Cancer in the state of Rio Grande do Norte: an ecological study Cáncer Oral en el estado de Río Grande do Norte: un estudio ecológico

Clébio Jarlison Rego de Freitas • Graduação em Odontologia-UFRN • Mestre em Saúde da Família RENASF/UFRN • Cirurgião Dentista-Prefeitura Municipal de Apodi/RN•E-mail: jarlisonodonto@gmail.com

José Adailton da Silva • Graduação em Enfermagem-Universidade Estadual do Rio Grande do Norte-UERN • Mestre em Saúde da Família RENASF/UFRN • Doutor em Saúde Coletiva - UFRN • Professor Adjunto da UFRN/FACISA e dos Programas de Pós Graduação em Saúde da Família-UFRN e Pós Graduação em Gestão e Inovação em Saúde-UFRN • E-mail: Silva.adailton@ufrn.edu.br

Maria Helena Pires Araújo Barbosa • Graduação em Enfermagem-FARN • Mestre em Saúde Coletiva-UFRN • Professora Assistente da Universidade Federal do Pará Faculdade de Medicina-Campus Altamira • Doutoranda em Enfermagem-UFSC • E-mail: mariahelenapab@hotmail.com

Laianny Krizia Maia Pereira • Graduação em Enfermagem - UNIFIP/Centro Universitário • Mestre em Saúde da Família-RENASF/UFRN • Pesquisadora do LAIS / Facilitadora Pedagógica do PEPSUS-UFRN • E-mail: laiannykmp@hotmail.com

Autora responsável pela correspondência:

Laianny Krizia Maia Pereira • E-mail: laiannykmp@hotmail.com 


\section{RESUMO}

Introdução: As neoplasias malignas ocupam o segundo lugar em causa de mortalidade no Brasil. Embora o país detenha grande número de dentistas em relação aos outros países, estudos apontam que o diagnóstico é feito tardiamente, levando assim, o câncer de boca como o oitavo câncer mais letal. Objetivo: Descrever a epidemiologia do câncer bucal no estado do Rio Grande do Norte. Método: Estudo epidemiológico descritivo do tipo ecológico realizado a partir dos casos diagnosticados de câncer bucal nos anos de 2000 a 2014. Resultados: Foram notificados 1.198 casos, sendo 714 óbitos; a região da língua e base de língua foi a mais acometida (33,63\%), com predominância de $63,18 \%$ do sexo masculino. Conclusões: O câncer bucal está ganhando destaque e estudos como este poderão subsidiar a proposição de políticas públicas e o planejamento de ações mais efetivas, para o diagnóstico precoce e o tratamento nos estágios iniciais da doença.

Palavras-Chave: Câncer. Câncer Bucal. Epidemiologia. Vigilância em Saúde.

\section{ABSTRACT}

Introduction: Malignant neoplasms are the second leading cause of death in Brazil. Although the country has a large number of dentists compared to other countries, studies indicate that the diagnosis is made late, thus leading to oral cancer as the eighth most lethal cancer. Objective: To describe the epidemiology of oral cancer in the state of Rio Grande do Norte. Methods: A descriptive epidemiological study of the ecological type carried out from the diagnosed cases of oral cancer in the years 2000 to 2014. Results: A total of 1198 cases were reported, of which 714 were deaths; The region of the tongue and tongue base was the most affected (33.63\%), with a predominance of $63.18 \%$ of males. Conclusions: Oral cancer is gaining prominence and studies such as this may support the proposal of public policies and the planning of more effective actions for the early diagnosis and treatment in the early stages of the disease.

Keywords: Cancer. Oral cancer. Epidemiology. Health Surveillance.

\section{RESUMEN}

Introducción: las neoplasias malignas son la segunda causa de mortalidad en Brasil. Aunque el país tiene una gran cantidad de dentistas en relación con otros países, los estudios muestran que el diagnóstico se hace tarde, por lo qu el cáncer de boca es el octavo cáncer más letal. Objetivo: Describir la epidemiología del cáncer oral en el estado de Rio Grande do Norte. Método: Estudio epidemiológico descriptivo del tipo ecológico realizado en base a los casos diagnosticados de cáncer oral en los años 2000 2014. Resultados: se reportaron 1.198 casos, de los cuales 714 fueron muertes; la región de la lengua y la base de la lengua fue la más afectada (33,63\%), con un rredominio del $63,18 \%$ de varones. Conclusiones: el cáncer oral está ganando rotagonismo y estudios como este pueden respaldar la propuesta de políticas úblicas y la planificación de acciones más efectivas para el diagnóstico y tratamiento mpranos en las primeras etapas de la enfermedad.

bras clave: Cáncer; Cáncer oral; Epidemiología; Vigilancia de la salud. 


\section{Introdução}

As neoplasias malignas já ocupam o segundo lugar em causa de mortalidade no Brasil ${ }^{1}$. Em 2030 o câncer deverá ser a principal causa de morte em nosso país, aumentando os custos de novas tecnologias para seu tratamento, levando a um sério desafio à saúde pública².

Dentre os inúmeros tipos de câncer, existe o grupo do câncer bucal ou da cavidade oral, que consiste em todas as neoplasias malignas que tenham sua localização nas regiões que compõe a boca: língua, gengiva, lábios, mucosa jugal, assoalho bucal e palato duro. A importância epidemiológica dessa doença é refletiva pela incidência estimada anual de câncer bucal que é de 324.398 casos no mundo, sendo estimado um aumento para 363.626 casos para o ano de 2020, com mortalidade estimada em 157 mil óbitos em $2015^{3}$.

No cenário brasileiro, para o biênio 2018-2019, o Instituto Nacional de Câncer (INCA) aponta a ocorrência de 600 mil casos novos para cada ano, sendo esperada a ocorrência de 11.200 casos novos de câncer da cavidade bucal em homens e 3.500 em mulheres. As Regiões Sudeste e Sul são as que apresentam as maiores incidências respectivamente, com o Nordeste Brasileiro na terceira posição. Ressalta-se que na Região Nordeste, o câncer bucal é o quinto mais frequente em homens e o décimo primeiro mais frequente em mulheres, com o Rio Grande do Norte ocupando a quinta posição na estimativa de casos para 2020, ficando abaixo dos estados da Bahia, Ceará, Pernambuco e Paraiba ${ }^{4}$.

Diversos fatores de risco estão relacionados ao desenvolvimento do câncer bucal. Contudo, o tabagismo e o consumo de álcool são reconhecidamente relacionados ao câncer bucal, e outros tipos de câncer como de faringe, esôfago e fígado. A exposição solar também está bastante relacionada aos casos de câncer de lábio, sendo o lábio inferior o mais acometido ${ }^{1,5}$.

Considerando que o Cirurgião-Dentista é o profissional capacitado para atuar enfermidades da região orofacial e sua presença na Estratégia saúde da Família se necessária para a realização de medidas preventivas e de diagnóstico, ${ }^{1}$ e embora o il detenha $19 \%$ dos cirurgiões dentistas do mundo ${ }^{6}$ e a sua inserção na ESF tenha 
crescido bastante com o programa Brasil Sorridente, estudos apontam que o diagnóstico ainda é feito tardiamente, ${ }^{1,7,8}$ levando assim, as neoplasias malignas como a segunda causa de mortalidade no Rio grande do Norte, e mais especificamente, o câncer de boca como o oitavo lugar no ranking dos cânceres mais letais 9 .

A eleição do enfrentamento do câncer bucal é uma prioridade para a saúde pública brasileira e, por consequência do estado Rio Grande do Norte (RN). Desta forma, se faz necessário o desenvolvimento de estudos que apontem a situação epidemiológica do câncer bucal. Diante do que foi exposto, o objetivo deste estudo é descrever o câncer bucal no Rio Grande do Norte, no período de 2000 a 2014.

\section{Metodologia}

Trata-se de um estudo epidemiológico descritivo, do tipo ecológico, realizado a partir dos casos diagnosticados de câncer bucal nos anos de 2000 a 2014 no Rio Grande do Norte.

A pesquisa foi realizada no Estado do Rio Grande do Norte, ao qual apresenta área de $52.796,79 \mathrm{~km}^{2}$, representando $3,41 \%$ da região nordeste e $0,62 \%$ de todo o território brasileiro. Segundo o Instituto Brasileiro de Geografia e Estatística (IBGE), sua população foi estimada em 3.474.998 habitantes para o ano de 2016, possuindo 167 municípios divididos em oito regiões de saúde.

Foram incluídos no estudo os casos de câncer bucal diagnosticados no estado do Rio Grande do Norte, registrados no Instituto Nacional do Câncer (INCA), no Registros Hospitalares de Câncer (RHC), bem como casos que foram a obtido registrados no Atlas de Mortalidade por Câncer, ambos disponibilizados no sítio eletrônico do INCA.

A base de dados foi construída de acordo com as seguintes variáveis: ocalização primária do tumor (língua, gengiva, lábios, mucosa jugal, assoalho bucal e alato duro), faixa etária (0-4 anos, 5-9 anos, 10- 14 anos, 15-19 anos, 20-29 anos, 30-39

nos, 40-49 anos, 50-59 anos, 60-69 anos, 70-79 anos e 80 anos ou mais) e sexo asculino e feminino), e uso de álcool ou tabaco (ex-usuário, usuário, não-usuário). 
Os dados foram obtidos de fonte secundária, sob domínio público no endereço eletrônico do INCA, não possuindo a identificação nominal dos indivíduos.

Para a análise dos dados foram utilizados os softwares Statistical Package for the Social Science (SPSS) versão 22.0 e o Microsoft Excel versão 2016 para tabulação e tratamento dos dados. A análise dos dados utilizou a estatística descritiva por frequência simples e distribuição percentual, e coeficiente de mortalidade bruta anual, ajustada, prevalência e letalidade, no período estudado

As taxas de mortalidade apresentadas foram retiradas diretamente da fonte de dados secundária e foram calculados pelo total de casos em determinado período/total da população do período X100.000. O ajuste por idade foi realizado pela seguinte equação ${ }^{4}$.

Taxa ajustada por idade $=\Sigma$ (taxa especifica por idade) $x$ (população padrão mundial na faixa etária/ $\Sigma$ População padrão mundial.

Após esse processo, foi feita a interpretação articulando o conhecimento apreendido com a leitura crítica do referencial bibliográfico trabalhado, possibilitando a comparação com teorias já existentes e a formulação de novas proposições.

Todo o estudo foi realizado com base nos princípios da ética e bioética, estabelecidos pela resolução n ${ }^{\circ} 466$ de 12 de dezembro de 2012 do Conselho Nacional de Saúde, sendo o mesmo dispensado pelo Comitê de Ética em Pesquisa por trabalhar com dados públicos e de livre acesso a população. A pesquisa está sujeita ao mínimo de risco possível, considerando que pode haver tão somente constrangimento, caso algum dado possa revelar fragilidade do estado em relação ao serviço de saúde ofertados.

\section{esultados}

No estado do Rio Grande do Norte, no período considerado, foram notificados 98 casos de câncer de boca, com a ocorrência de 714 óbitos como causa básica, 
conforme ilustrado na Tabela 01. A prevalência no período foi de 37,81 casos para cada 100.000 habitantes e a taxa de letalidade chegou a 59,59\% dos casos.

Tabela 01. Casos diagnosticados e Taxa de mortalidade de câncer bucal ajustadas por ano por 100.000 habitantes. Rio Grande do Norte, 2000 - 2014. Natal-RN, 2017.

\begin{tabular}{ccccc}
\hline Ano & $\begin{array}{c}\text { Casos novos } \\
\text { diagnosticados }\end{array}$ & Óbitos & $\begin{array}{c}\text { Taxa bruta } \\
\mathbf{( \% )}\end{array}$ & Taxa ajustada (\%) \\
\hline 2000 & 16 & 22 & 0,79 & 0,75 \\
2001 & 38 & 26 & 0,92 & 0,91 \\
2002 & 31 & 30 & 1,05 & 1,03 \\
2003 & 41 & 33 & 1,14 & 1,1 \\
2004 & 38 & 39 & 1,33 & 1,26 \\
2005 & 101 & 54 & 1,8 & 1,72 \\
2006 & 42 & 51 & 1,68 & 1,56 \\
2007 & 31 & 43 & 1,39 & 1,27 \\
2008 & 51 & 50 & 1,61 & 1,42 \\
2009 & 99 & 58 & 1,85 & 1,59 \\
2010 & 126 & 58 & 1,83 & 1,41 \\
2011 & 168 & 71 & 2,22 & 1,7 \\
2012 & 129 & 66 & 2,04 & 1,54 \\
2013 & 144 & 54 & 1,67 & 1,3 \\
2014 & 143 & 59 & 1,83 & 1,48 \\
Total & 1198 & 714 & - & - \\
\hline
\end{tabular}

Fonte: Informações do Registro Hospitalar de Câncer - Tabulador Hospitalar, Atlas online de mortalidade. INCA - Ministério da Saúde, 2017.

Em relação à localização primária, observou-se predomínio da língua e base de língua com 403 (33,63\%) indivíduos, seguido pelo palato com 223 (18,61\%), lábio com $219(18,28 \%)$, outras partes da boca com $211(17,61 \%)$, assoalho da boca com 123 $(10,26 \%)$ e gengiva com 19 (1,58\%). O percentual em que ocorreu maior variação foram nos casos de câncer de língua e câncer de lábio. O câncer de língua apresentou valores que variaram entre $8(21,05 \%)$ indivíduos, no ano de 2001, a $46(31,16 \%)$ indivíduos em 2014. Já o câncer localizado no lábio teve 10 (26,31\%) indivíduos em 2001 e 16 (11,18\%) divíduos no ano de 2014.

Quanto ao sexo, verificou-se que 757 (63,18\%) dos sujeitos eram do sexo sculino, com proporção de homens para mulheres de 1,7:1. A predominância dos 
casos no sexo masculino sobre o sexo feminino foi verificada em todos os anos do estudo. Em todos os sítios de localização, apenas na região do palato ocorreu mais casos em mulheres $(57,39 \%)$ do que em homens $(42,61 \%)$. Nos demais, o número de casos foi mais que o dobro em indivíduos do sexo masculino, chegando ao triplo na região de base de língua.

O maior número de casos de câncer bucal no estado do Rio Grande do Norte ocorreu na faixa etária acima de 50 anos, com ênfase no grupo de indivíduos de 60 a 69 anos. Importante destacar que 83,05\% (995 sujeitos) dos casos ocorreram em indivíduos com idade acima de cinquenta anos. O número de casos em indivíduos menores de quinze anos perfez um total de apenas 0,52\% (6 indivíduos) com predomínio na faixa etária de cinco a nove anos. No período analisado, a taxa de mortalidade foi de 1,54\%, no qual 58,12\% (430 indivíduos) dos óbitos registrados pertenciam ao sexo masculino, sendo as localizações primárias com maior taxa de mortalidade as outras partes da boca $(0,77 \%)$, língua e base de língua (0,53\%). A tabela 02 mostra a taxa de mortalidade ajustada por faixa etária e sexo.

Tabela 02. Taxa de mortalidade ajustada por faixa etária e sexo, por 100.000 habitantes. Rio Grande do Norte, 2000 - 2014. Natal-RN,, 2017.

\begin{tabular}{ccc}
\hline Faixa-etária & Sexo Masculino & Sexo feminino \\
\hline $\mathbf{0 0}$ a $\mathbf{0 4}$ & 0,1 & 0,05 \\
$\mathbf{0 5}$ a $\mathbf{0 9}$ & 0 & 0 \\
$\mathbf{1 0}$ a $\mathbf{1 4}$ & 0 & 0 \\
$\mathbf{1 5}$ a $\mathbf{1 9}$ & 0 & 0 \\
$\mathbf{2 0}$ a $\mathbf{2 9}$ & 0,1 & 0,08 \\
$\mathbf{3 0}$ a 39 & 0,4 & 0,09 \\
$\mathbf{4 0}$ a 49 & 2,54 & 0,48 \\
$\mathbf{5 0}$ a $\mathbf{5 9}$ & 7,57 & 1,53 \\
$\mathbf{6 0}$ a $\mathbf{6 9}$ & 9,97 & 3,52 \\
$\mathbf{7 0}$ a 79 & 13,13 & 10,63 \\
$\mathbf{8 0}$ ou mais & 24,31 & 31,54 \\
\hline
\end{tabular}

Fonte: Informações do Registro Hospitalar de Câncer - Tabulador Hospitalar, Atlas on-line de mortalidade. INCA - Ministério da Saúde, 2017.

As tabelas 03 e 04 apresentam percentual de casos e sua localização e o consumo bebidas alcóolicas ou o uso do tabaco no período de 2000 a 2014. Importante 
destacar o alto número de casos sem informação, o que pode sugerir a existência de subnotificação e contribuir para o mascaramento de informações.

Tabela 03. Percentual de casos segundo a localização primária em relação ao consumo de bebida alcoólica. Rio Grande do Norte, 2000 - 2014. Natal-RN, 2017.

\begin{tabular}{cccccc}
\hline Localização primária & $\begin{array}{c}\text { Ex- } \\
\text { consumidor }\end{array}$ & Nunca & $\begin{array}{c}\text { Sem } \\
\text { Informação }\end{array}$ & Sim & Total \\
\hline Lábio & 3,20 & 31,96 & 49,32 & 15,53 & 18,28 \\
Base da língua & 13,10 & 17,86 & 15,48 & 53,57 & 7,01 \\
Língua & 10,34 & 20,69 & 23,20 & 45,77 & 26,63 \\
Gengiva & 10,53 & 26,32 & 21,05 & 42,11 & 1,59 \\
Assoalho da boca & 8,13 & 24,39 & 16,26 & 51,22 & 10,27 \\
Palato & 7,62 & 36,32 & 29,15 & 26,91 & 18,61 \\
Outras partes da boca e das & 9,00 & 29,38 & 18,01 & 43,60 & 17,61 \\
não especificadas & & $\mathbf{2 7 , 4 6}$ & $\mathbf{2 6 , 8 8}$ & $\mathbf{3 7 , 4 0}$ & $\mathbf{1 0 0 , 0 0}$ \\
\hline Total & $\mathbf{8 , 2 6}$ & $\mathbf{2 6 0}$
\end{tabular}

Fonte: Informações do Registro Hospitalar de Câncer - Tabulador Hospitalar. INCA, 2017.

Tabela 04. Percentual de casos segundo a localização primária em relação ao uso do tabaco. Rio Grande do Norte, 2000 - 2014. Natal-RN, 2017.

\begin{tabular}{ccccccc}
\hline $\begin{array}{c}\text { Localização } \\
\text { primária }\end{array}$ & $\begin{array}{c}\text { Ex- } \\
\text { fumante }\end{array}$ & $\begin{array}{c}\text { Não se } \\
\text { aplica }\end{array}$ & Nunca & $\begin{array}{c}\text { Sem } \\
\text { Informação }\end{array}$ & Sim & Total \\
\hline Lábio & 7,76 & 0,00 & 22,37 & 35,16 & 34,70 & 18,28 \\
Base da língua & 21,43 & 0,00 & 11,90 & 5,95 & 60,71 & 7,01 \\
Língua & 14,73 & 0,31 & 10,97 & 14,73 & 59,25 & 26,63 \\
Gengiva & 10,53 & 0,00 & 10,53 & 10,53 & 68,42 & 1,59 \\
Assoalho da boca & 16,26 & 0,00 & 7,32 & 10,57 & 65,85 & 10,27 \\
Palato & 11,21 & 0,00 & 6,73 & 21,52 & 60,54 & 18,61 \\
$\begin{array}{c}\text { Outras partes da } \\
\text { boca e das não }\end{array}$ & 20,85 & 0,00 & 12,80 & 9,48 & 56,87 & 17,61 \\
especificadas & & & & & & \\
Total & $\mathbf{1 4 , 4 4}$ & $\mathbf{0 , 0 8}$ & $\mathbf{1 2 , 2 7}$ & $\mathbf{1 7 , 7 0}$ & $\mathbf{5 5 , 5 1}$ & $\mathbf{1 0 0 , 0 0}$ \\
\hline
\end{tabular}

Fonte: Informações do Registro Hospitalar de Câncer - Tabulador Hospitalar. INCA, 2017.

\section{Discussão}

A incidência de neoplasias malignas da cavidade bucal difere bastante entre as regiões do Brasil, isto pode ser associado as diferenças na exposição aos fatores de risco como: exposição solar, consumo de tabaco e álcool e dieta, além da carga genética ${ }^{10}$.

Nas estimativas para 2020, o câncer da cavidade bucal em homens é o quinto mais frequente nas Regiões Sudeste (13,58/100 mil), Centro-Oeste (8,94/100 mil) e 
Nordeste (7,65/100 mil). Nas Regiões Sul (13,32/100 mil) e Norte (3,80/100 mil), ocupa a sexta posição. Para as mulheres, é o décimo primeiro mais frequente na Região Nordeste (3,75/100 mil) e o décimo segundo na Região Norte (1,69/100 mil). Já nas Regiões Sudeste (4,12/100 mil) e Centro-Oeste (2,90/100 mil), ocupa a décima terceira posição. Na Região Sul (4,08/100 mil), ocupa a décima quarta posição ${ }^{4}$.

O Nordeste brasileiro possui como atividade principal a agricultura e pecuária. Questões sociais, comportamentais, econômicas e culturais estão intimamente ligadas à adoção de comportamentos de risco que influenciam na incidência desse tipo de câncer ${ }^{11}$. Entres os estados da região Nordeste, estimativas para 2020 coloca o Rio Grande do Norte na terceira posição em novos casos entre homens (9,82/100.000 habitantes) e em primeiro lugar entre mulheres (7,07/100.000 habitantes) Em relação ao sexo feminino em todo o País, o estado só perde a estimativa para Santa Catarina. Em um estudo realizado por Freire ${ }^{12}$, as capitais nordestinas Salvador, Natal e Recife apresentaram as maiores taxas de incidência do câncer de boca em contraste com São Luís e Teresina que obtiveram índices mais baixos

O câncer bucal não apresenta uma incidência tão expressiva como o câncer próstata e de mama no estado do Rio Grande do Norte. Porém, apesar do baixo número de casos, a alta taxa de letalidade dos casos diagnosticados no período é preocupante, o que pode indicar um diagnóstico feito tardiamente, visto que o diagnóstico precoce está diretamente ligado a sobrevida do indivíduo. Contrapondo a isso, os avanços nas técnicas usadas no tratamento, também, podem influenciar na qualidade de vida do paciente e impossibilitando possíveis recidivas que finalizem em óbito ${ }^{13}$.

O diagnóstico realizado em estágios tardios acarreta um pior prognóstico e diminuição da taxa de sobrevida. Esse atraso estaria associado a três situações, (i) atraso do paciente na percepção do primeiro sinal, (ii) atraso no agendamento para consulta com o profissional e (iii) o atraso do período entre a primeira consulta até o xame patológico definitivo ${ }^{8}$.

A rede de atenção em saúde bucal no estado é composta pela atenção primária cundária. O diagnóstico é realizado na atenção secundária, que atualmente é 
composta por 29 Centro de Especialidades Odontológicas e existem duas universidades públicas com cursos de odontologia que também realizam o diagnóstico de lesões. A distribuição irregular entre as cidades dificulta o acesso aos serviços de diagnóstico bucal, podendo ser uma barreira contra o tempo na realização de um diagnóstico precoce e início do tratamento. A cobertura da atenção secundária em saúde bucal no estado apresenta uma distribuição desigual, uma vez que há variação de $8 \%$ a $80 \%$ entre as regiões de saúde ${ }^{14}$. Esta desigualdade na distribuição resulta em acesso desigual aos serviços de saúde bucal nas diferentes regiões de saúde do estado. Isso implica em um obstáculo a ser transposto pelos profissionais da saúde bucal com vistas ao diagnóstico precoce.

Dentre os tipos de neoplasias malignas presentes na boca, 90\% são do tipo carcinoma epidermoide ${ }^{10}$. No Rio Grande do Norte, esse tipo esteve presente em 1084 casos $(90,48 \%)$. Tal dado é muito preocupante pois, o carcinoma epidermóide oral exibe elevadas taxas de mortalidade, mesmo com o avanço terapêutico. Pode-se atribuir esse fato principalmente à resposta variada ao tratamento e ao diagnóstico $\operatorname{tardio}^{14}$.

O número de casos vem crescendo a cada ano, levando o câncer bucal a uma posição preocupante. Apesar do maior acometimento ser no sexo masculino em todos os anos do estudo, concordando com a literatura,15,16 a diferença entre sexos está diminuindo devido a exposição cada vez mais frequente das mulheres aos principais carcinógenos, como álcool e fumo ${ }^{17}$.

Diversos autores confirmam que a faixa etária mais acometida é de 60 a 69 anos e que o acometimento de crianças é muito raro. A maior prevalência em idosos pode ser devido ao envelhecimento celular, diminuindo assim a capacidade de se recuperar ${ }^{18,19}$. Localizações com maior prevalência vistas neste estudo também foram relatados por outros autores ${ }^{20,21}$. O lábio inferior está anatomicamente mais exposto as radiações solares, fator predisponente para o surgimento da doença ${ }^{22}$.

Quanto aos fatores predisponentes que foram apresentados nas tabelas 02 e 03 , dados corroboram com o estudo realizado por Andrade, Santos e Oliveira ${ }^{23}$, onde a 
maioria dos casos encontrados eram em indivíduos fumantes e ex-fumantes ou que fazem uso de bebidas alcoólicas ${ }^{23}$.

O uso de tabaco está associado a 90\% do desenvolvimento da doença, por aumentar a temperatura dentro da boca, e possuir mais de 50 substâncias com potencial carcinogênico. Também é importante destacar a evidente a atuação sinérgica do tabaco e do álcool, sendo essa atuação dose-dependente ${ }^{3,22,24}$.

O álcool em contato com a mucosa parece agir como um solvente, facilitando a passagem de carcinógenos através das membranas celulares e alterando metabolismo intracelular das células epiteliais com as quais ele entra em contato. Seu consumo também eleva a atividade metabólica do fígado, podendo, por conseguinte, ativar substâncias carcinogênicas ${ }^{22}$. O álcool presente nos enxaguantes bucais, que pode chegar à concentração de 27\%, agride quimicamente as células na mesma proporção que a bebida alcoólica, se for usado várias vezes ao dia ${ }^{25}$.

Dessa forma, uma vez que o Rio Grande do Norte apresenta a $2^{\mathrm{a}}$ maior incidência de câncer bucal em mulheres e a $14^{\mathrm{a}}$ em homens entre os estados brasileiros, faz-se necessário incrementar ações e serviços que reduzam a mortalidade relacionada à doença no estado ${ }^{1}$. É importante reconhecer o papel do Cirurgião-Dentista como ator principal na realização do diagnóstico precoce e sua atuação frente a prevenção e promoção da saúde quando ele está inserido na atenção primária.

Os profissionais da atenção primária possuem um papel de destaque nas ações de prevenção e diagnóstico precoce. As atividades de promoção da saúde na prevenção do câncer bucal devem se voltadas para o tabagismo e consumo consciente de álcool, principais fatores relacionados ao câncer bucal. E as salas de espera e recepções constituem ambientes para a disseminação de informações sobre o assunto. A integração da atenção primária e secundária a uma rede de diagnóstico de câncer bucal favorece a realização de biópsias, a identificação de lesões malignas e o liagnóstico precoce dessas lesões ${ }^{1,22}$. 


\section{Conclusões}

Neste estudo procurou-se destacar a epidemiologia do câncer bucal no estado do RN. Para isso demonstrou dados como a prevalência, a letalidade e a taxa de mortalidade (bruta e ajustada) por câncer bucal. Apresentou também os dados acerca da mortalidade ajustada conforme o sexo, assim como dados referentes à localização primária e o consumo de bebida alcóolica e uso tabaco.

Na população investigada o câncer bucal foi predominante em homens com a faixa etária de 60 a 69 anos. A língua e a base da língua foi a localização primária predominante. Com relação ao consumo de bebida e uso de tabaco observou-se um alto número de casos sem informação Esta ausência de dados pode indicar um viés de informação e há possibilidade de subregistro em virtude da relevância desses fatores de risco para o desenvolvimento desse câncer.

O câncer bucal é sensível à atenção básica, mas o acesso e a qualidade das ações preventivas com vistas ao diagnóstico precoce perpassam pelos serviços de saúde bucal e pela assistência prestada pelos profissionais de saúde. A desigualdade quanto a disponibilidade de serviços de saúde bucal nas diferentes regiões de saúde do RN pressupõe que, para o adequado enfrentamento do câncer bucal no estado, há necessidade de melhorias quanto aos entraves para o acesso, a cobertura e a articulação entre os serviços da rede de atenção à saúde bucal no RN.

Por fim, este estudo poderá contribuir para o planejamento de ações voltadas para o diagnóstico precoce e o tratamento nos estágios iniciais da doença, de forma a diminuir o elevado percentil de mortalidade dos casos observados no estudo.

\section{Referências}

Torres-Pereira CC, Angelin-Dias A, Melo NS, Lemos Jr. CA, Oliveira EMF. bordagem do câncer de boca: uma estratégia para os níveis primário e secundário atenção em saúde. Cad Saúde Pública 2012 [acesso em 15 abr 2018], 28 Suppl:S30Disponível em: <http://www.scielo.br/scielo.php?script=sci_arttext\&pid=S0102X2012001300005> 
2. Barbosa IR, Costa ICC, Bernal MM, Souza DLB.Tendência das taxas de mortalidade pelas dez principais causas de óbitos por câncer no Brasil, 1996-2012. Rev. Ciênc. Plur 2016 [acesso em 02 nov 2019], 2(1):3-16. Disponível em: <https://periodicos.ufrn.br/rcp/article/view/8886/7114>.

3. Global Cacer Observatory [homepage na internet]. Cancer incidence, mortality and prevalence worldwide [acesso em 28 set 2016]. Disponível em: http://gco.iarc.fr/

4. Ministério da Saúde. 2020. Instituto Nacional de Câncer. Estimativa: incidência de câncer no Brasil. [homepage na Internet]. [Acesso em 29 de março de 2020]. Disponível em: http://www1.inca.gov.br

5. Vasconcelos RM, Trindade JSO, Almeida ICP, Silva RJC, Morais MLSA. Perfil clínico-epidemiológico de câncer de boca em idosos. Rev Gestão \& Saúde [periódicos na internet]. 2013 Mar [acesso em 15 fev 2017];1984-91. Disponível em: file:/ / C:/Users/User/Downloads/214-Texto\%20do\%20artigo-438-1-10$20170807 \% 20(1) \cdot p d f$

6. Conselho Federal de Odontologia [homepage na Internet]. Brasil é o país com o maior número de dentistas [acesso em 14 jan 2017]. Disponível em:

http:/ / cfo.org.br/sem-categoria/brasil-e-o-pais-com-o-maior-numero-de-dentistas/

7. Gómez I, Seoane J, Varela-Centelles P, Diz P, Takkouche B. Is diagnostic delay related to advanced-stage oral cancer? A meta-analysis. Eur J Oral Sci [periódicos na internet]. 2009 Out [acesso em 14 jan 2017];117(5):541-6. Disponível em: https://onlinelibrary.wiley.com/doi/abs/10.1111/j.1600-0722.2009.00672.x

8. Gómez I, Warnakulasuriya S, Varela-Centelles P, López-Jornet P, SuárezCunqueiro M, Diz-Dios P, et al. Is early diagnosis of oral cancer a feasible objective? Who is to blame for diagnostic delay?. Oral Dis [periódicos na internet]. 2010 Mai [acesso em 14 jan 2017];16(4):333-42. Disponível em:

https://onlinelibrary.wiley.com/doi/abs/10.1111/j.1601-0825.2009.01642.x

9. Ministério da Saúde [homepage na internet]. Sistema de informações sobre Mortalidade [acesso em 12 mar 2017]. Disponível em:

<http://tabnet.datasus.gov.br/cgi/tabcgi.exe?sim/cnv/obt10uf.def>

10. Carli ML, Santos SL, Pereira AAC, Hanemann JAC. Características Clínicas, Epidemiológicas e Microscópicas do Câmcer Bucal Diagnosticado na Universidade Federal de Alfenas. Rev Bras Cancerol 2009;55(3):205-211.

Barreto RC, Paiva MAF, Soares MSM, Pereira GAS. Prevalência de Câncer Bucal Mesorregiões Paraibanas. R bras ci Saúde 2006; 10(1):9-16. 
12. Freire JCP, Oliveira CR, Câmara AO, Valença AMG, Santiango BM. Incidência de neoplasias malignas orais nas regiões brasileiras e capitais do Nordeste. Arch Health Invest (2017) 6(11):535-539.

13. Bonafante GMS, Machado CJ, Souza PEA, Andrade EIG, Acurcio FA, Cherchiglia ML. Sobrevida de cinco anos e fatores associados ao câncer de boca para pacientes em tratamento oncológico ambulatorial pelo Sistema Único de Saúde, Brasil. Cad Saude Publica [periódicos na internet] 2014 [acesso em 14 jan 2017] 30(5):983-97. Disponível em: https://doi.org/10.1590/0102-311X00182712

14. Silva AP, Dutra LC, Martins YVM, Araújo HSP, Seabra EJG. Cobertura da atenção secundária no Rio Grande do Norte (RN). Rev Abeno 2015;15(2):65-73.

15. Volkweis MR, Blois MC, Zanin R, Zamboni R. Perfil Epidemiológico dos Pacientes com Câncer Bucal em um CEO. Rev Cir Traumatol Buco-Maxilo-Fac. 2014; 14(2):63-70.

16. Melo AUC, Albuquerque Junior RLC, Melo MFB, Ribeiro CF, Santos TS, Gomes ACA. Análise das estimativas de incidência de câncer de boca no Brasil e em Sergipe (2000 - 2010). Odontol Clín-Cient 2012; 11(1):65-70.

17. Andrade JOM, Santos CAST, Oliveira MC. Fatores associados ao câncer de boca: um estudo de casocontrole em uma população do Nordeste do Brasil. Rev Bras Epidemiol 2015; 18(4):894-905.

18. Amorim-Filho FS, Andadre-Sobrinho J, Rapoport A, Novo NF, Juliano Y. Estudo clínico-epidemiológico do carcinoma epidermóide da base da língua. Rev Bras Otorrinolaringol [periódicos na internet] 2003 [acesso em 14 jan 2017]; 69(2):175-9. Disponível em: http://www.scielo.br/pdf/rboto/v69n2/15621.pdf

19. Sawair Fa, Irwin CR, Gordon DJ, Leonar AG, Stephenson M, Napier SS. Invasive frent grading: reliability and usefulness in the management of oral squamous cell carcinoma. J Oral Pathol Med 2003 Jan;32(1):1-9.

20. Costa ALL, Araujo-Júnior RF, Ramos CCF. Correlação entre a classificação clínica TNM e as característicass histológicass de malignidade do carcinoma epidermóide oral. Rev Bras Otorrinolaringol [periódicos na internet]. 2005 Mar [acesso em 14 jan 2017];71(2):181-187. Disponível em: http://www.scielo.br/pdf/rboto/v71n2/a11v71n2.pdf

21. Garzino-Demo P, Dell' Acqua A, Dalmasso P, Fasolis M, La Terra-Maggiore GM, amiere $\mathrm{G}$, et al. Clinicopathological parameeters and outcome of 245 patients peradted for oral squamous cell carcinoma. J Craniomaxillofac Surf 2006 ;34(6):344-350.

Domingos PAS, Passalacqua MLC, Oliveira ALBM. Câncer bucal: Um problema aúde pública. Rev Odontol Univ Cid São Paulo 2014 Jan;26(1):46-52. 
23. Andrade JOM, Santos CAST, Oliveira MC. Fatores associados ao câncer de boca: um estudo de caso-controle em uma população do nordeste do Brasil. Rev Bras Epidemiol 2015; 18(4): 894-905. [acesso em 20 out 2019];71(2):181-187. Disponível em: <http://www.scielo.br/pdf/rbepid/v18n4/1980-5497-rbepid-18-04-00894.pdf>

24.Scheidt JHG, Yurgel LS, Cherubini K, Figueiredo MAZ, Salum FG. Características do carcinoma bucal de células escamosas em usuários ou não usuários de tabaco e álcool. Rev Odonto Ciência 2012;27(1):69-73.

25. Buelvas AR, Agudelo SAA. Gradiente social, envejecimiento y diagnóstico tardío del cáncer oral. Rev Fac Nac Salud Pública 2011 Set;29(3):320-8. 